Pak. j. sci. ind. res. Ser. B: biol. sci. 2020 63B(3) 163-168

\title{
Evaluation and Insilico Analysis of Genes Involved in Hypothyroidism
}

\author{
Ammara Masood*, Hira Mubeen and Nadia Iqbal \\ University of Central Punjab, Lahore, 1-Khayaban-e-Jinnah Road, Johar Town, Lahore, Pakistan
}

(received July 31, 2018; revised December 21, 2018; accepted December 24, 2018)

\begin{abstract}
To evaluate the expression of genes that involved in hypothyroidism by multiple alignment of nucleotide sequences of genes study the functional unit of genes that involve in production of thyroid hormones. The thyroid gland is part of endocrine gland and regulates much vital body function (breathing, heart rate, body weight, body temperature also involve in development, growth, protein production and it also control the central and peripheral nervous system, muscle strength and menstrual cycle). Hypothyroidism is a condition in which thyroid gland does not produce enough thyroid hormone. To find the genetics information than by using bioinformatics tools several genes that were involved in hypothyroidism were identified but six genes TSHR, TPO, THRB, TG, IYD, DUOX2 and their motifs were studied in detail to understand the basic domains involved in this disease.
\end{abstract}

Keywords: thyroid gland, TSHR, TPO, THRB, TG, hypothyroidism, IYD, DUOX2 bioinformatics tools

\section{Introduction}

The thyroid gland is an endocrine gland which produces, release and store hormone into blood and hormone transferred to all the cells of body which affects the protein production and metabolic rate (Vetter et al., 2008). The production of thyroid hormone is controlled by thyroid-stimulating hormones (TSH) which are secreted by anterior pituitary gland. Thyroid stimulating hormone (TSH) activates the enzymes that release T4 and T3 from thyroglobulin. Mostly T4 as the primary hormone produced and released from the thyroid gland (Surks et al., 2004). TSH released and is stimulated by thyrotropin releasing hormone (TRH), which are liberated in pulsate way from the hypothalamus (Engum et al., 2002). When thyroid hormones are high, they provide negative feedback to thyrotropes and TRH than TSH production is repressed. When high levels of TSH, than negative feedback occur and repressed the TRH production (Pilo et al., 1990). Several diseases of thyroid are known but most common is hypothyroidism (Wekking et al., 2005).

Hypothyroidism is a defect of thyroid gland in which it produces sufficient amount of thyroid hormone to meet metabolic demands of the body. When hypothyroidism remains untreated than causes cognitive impairment, dyslipidemia, hypertension, neuromuscular dysfunction and infertility (Hollowell et al., 2002). There are two types of hypothyroidism primary and

*Author for correspondence;

E-mail: ammara.masood@ucp.edu.pk secondary. Primary hypothyroidism is defect of thyroid gland and decreases in production and release of thyroid hormones. Hormones pills are taken as a treatment of hypothyroidism. It is also important to take the right dose otherwise it can cause the primary hypothyroidism (Vetter et al., 2008). Secondary hypothyroidism occurs by disorder in pituitary gland and hypothalamus that resulting in decrease in TSH and also causes decreased in the secretion and production of thyroid hormones. Secondary hypothyroidism also known central hypothyroidism, and divided into secondary and tertiary hypothyroidism. When the pituitary gland is defected than it is called secondary and when in hypothalamus than known as tertiary hypothyroidism. Many disorders causes' secondary hypothyroidism and most commonly known causes are pituitary adenomas. For its treatment, surgery and radiotherapy are used (Hedberg et al., 1990). Endoscopic Thyroid Surgery is an endoscopic techniques as an approach to neck surgery and specifically thyroid surgery has been an novel method of eliminating the most common complaint postoperativelythe unsightly scar. The advantages of surgery include the magnification of the anatomy, enhanced illumination of the operative field.

If high TSH and low thyroxin level than mean that thyroid gland underactive. It also indicates that pituitary gland release thyroid stimulating hormone in high concentration that stimulates the thyroid to synthesized hormone (Goichot et al., 2015). Thyroid gland is the vital organ of human body and genetic basis of various diseases of thyroid glands are studied. 
Thyroid peroxidase (TPO) gene catalyase the reaction in which iodine is inserted into a protein called thyroglobulin. It's an analytical step in the synthesis of hormone. To perform their proper function, peroxidase should be present in the membrane of thyroid follicular cells. When peroxidase is not present in the cell membrane than peroxidase do not able to perform their function in the proper way (Andersson et al., 2005). Thyroid hormone receptors (TRs) are nuclear receptor and have effect for gene regulation by hormone (thyroxine (T4) or tri-iodothyronine T3). Thyroglobulin (TG) is a glycoprotein homodimer which are synthesized by thyroid. It works as a substrate production for the $\mathrm{T} 4$ and $\mathrm{T} 3$ and also stored iodinethyroid hormone in the inactive forms. From iodination site of endoplasmic reticulum thyroglobulin is secreted, than in the follicular lumenthyroxine is biosynthesized. Due to mutation, TG causes dyshormonogenesis of the thyroid gland, demonstrated as goiter, also associated with mild to severe congenital hypothyroidism (Andersson et al., 2005). Iodothyronine (IYD) deiodinase gene encodes a pretein that is an enzyme and the oxidative NADPHdependent deiodination of mono- and diiodotyrosine are catalyzed by that enzyme which are the halogenated by products of thyroid hormone production. Iodothyronine deiodinase are a subfamily of deiodinase enzyme and also have importance in the deactivation and activation of thyroid hormones. The precursor of 3,5,3'triiodothyronine (T3) and thyroxine (T4) is transferes into $\mathrm{T} 3$ by the activity of deiodinase. Triiodothyronin binds with a nuclear thyroid hormone receptor, influence the genes expression specifically in every vertebrate cell (Taurog and Howells, 2000). Dual oxidase is an enzyme of human body which is coded by a DUOX2 gene. The hDUOX1 is prominent in airway epithelial cells and hDUOX2 in the salivary glands and gastrointestinal tract (Kimura et al., 1989 and De Vijlder et al., 1988). In present study most common genes of hypothyroidism TSHR, TPO, THRB, IYD, TG, DUOX2 are studied.

\section{Materials and Methods}

National Center for Biotechnology Information (NCBI, National Institutes of Health, Bethesda, MD) was used for general purpose sequence similarity searches. NCBI gene tool (https://www.ncbi.nlm.nih.gov) used to search human genes of hypothyroidism like TSHR, TPO, THRB, IYD, TG, and DUOX-2. NCBI Gene tool was used for the collection of basic information of these genes. By using the NCBI nucleotide tool Nucleotide sequence of genes were collected in FASTA format.
Orthologous sequences of these genes were studied by using the OTTH, orthodisease and ensemble tool. The complete dataset of gene clusters and associated disease information was made available through an online searchable tool named OrthoDisease (http://orthodisease. cgb.ki.se/). Ensemble tool explain the genes that they have common ancestor and displayed in blue color in phylogenic tree (https://asia.ensembl.org/index.html). For find the general function of genes HGNG (OMIM) tool is used (https;//www.omim.org/entry/188450). For multiple alignment of the sequence of genes different tools were used. From Multialin "multialin.toulouse.inra. and Alibee multiple alignment tools (www.genebee.msu. $\mathrm{su} /$ services/malign_reduced.html) all six gene sequences were aligned. Conserved Doman database is also used to find the image of proteins of each gene (https://www. ncbi.nlm.nih.gov/structure/cdd/wrpsb.cgi).

\section{Results and Discussion}

For the multiple alignment second tool which is used are alibee mutiple alignment and results are given in Fig. 1. A phylogenetic tree is a branching diagram or "tree" showing the inferred evolutionary relationships among biological species about their phylogeny, based upon similarities and differences in their physical or genetic characteristics. Genes BC054883.1, DQ011222.1 and AF267981.1. They show close similarity to each other in phylogeny. KJ900706.1 and KJ897710.1 show close similarity in phylogenetic tree. Fig. 2A and B. Thyroid hormones have significant role, in homeostasis in mammals, growth and development. Nucleotide scoring distance matrix shown in Fig. 3 are used in phylogeny as non-parametric distance methods and were originally applied to phenetic data using a matrix

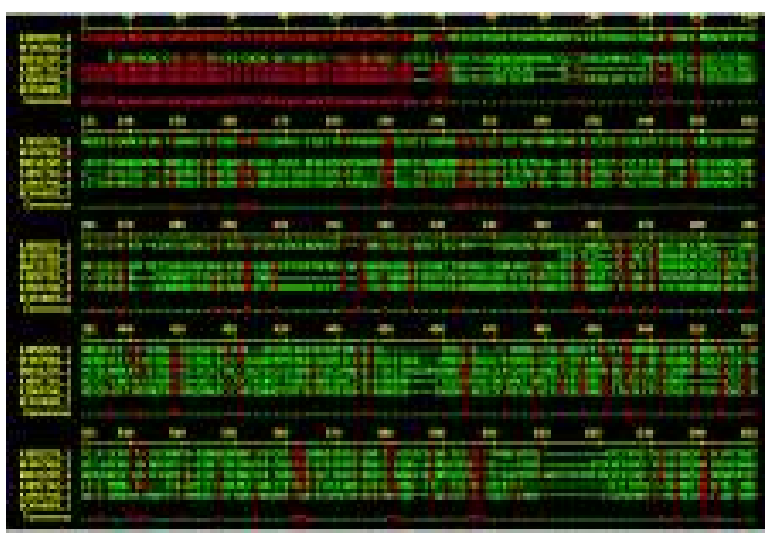

Fig. 1. The image of multiple allignment of genes from multialin tool. 
of pairwise distances. These distances are then reconciled to produce a phylogenetic tree. They are also invlove in the regulation of heart beat, triglyceride levels, cholesterol, and metabolic rate and also have effects on mood. In the expression of thyroid hormones receptors two separate genes are involved. Alpha and beta are the two separate genes in the human and both genes by using different promoter and by splicing produce two isoforms of receptors (Bakker et al., 2000). Thyroid receptor alpha (Tralpha) perform their function in the regulation of heart rate and rhythm. Two active domain were also present in most of the genes, central well conserved DNA binding domain (DBD) and a C-terminal ligand binding domain (Cangul et al., 2013). Genes retrieved from HGNC of hypothyroidism have these domain having consensus-sequence:

“ACGGGTTCCCACTGCCCCCGGTCCGGGAGG TGACAAGACATGTCA"“CTTCCTGCAGAAAAC AC",,GTCATTTCCGGAAGGTCCTGAACAAGGG”, “AAAGTTGGCATTATAAGAAAGCATTGCTTAT CAATTTGTTG”
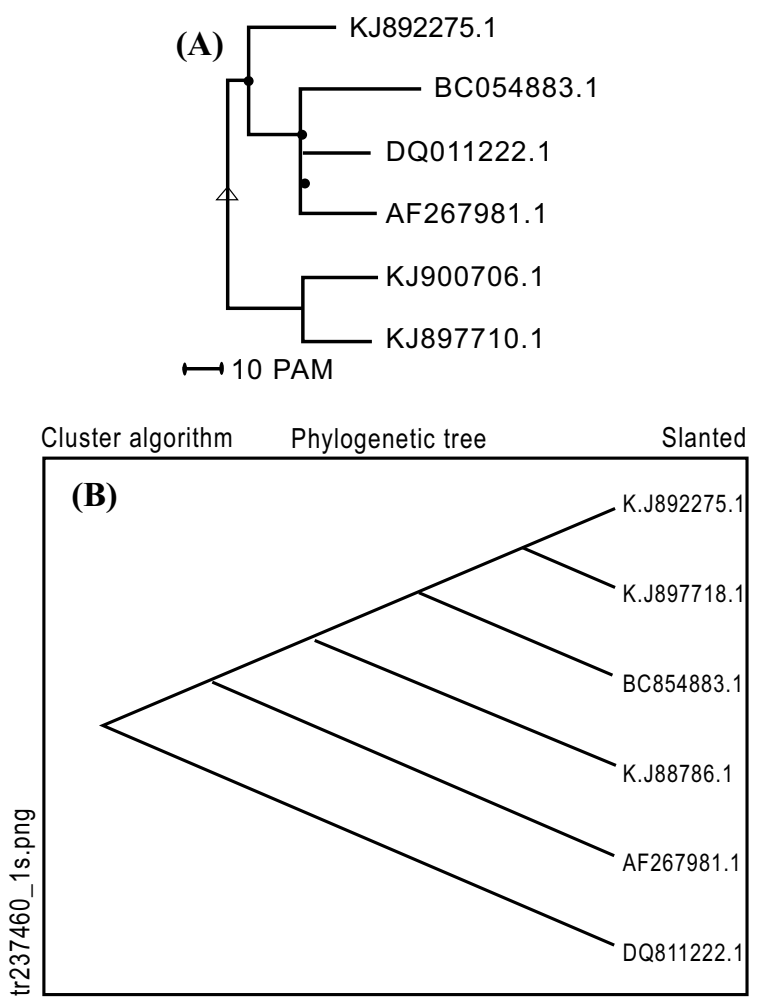

Fig. 2. (A) Phylogenetic tree of all six hypothyroid gene; (B) Cladogram of genes.
Conserved repeats between two species were those with a length of 5 or longer in an equivalent position in the two sequences these motifs are involved in functional units of hypothyroid genes. Some are glutamine tandem repeats mapped on the aligned sequences. The dataset included different known CAG-expansion disease genes. Gene Protein 3D folding picture gives insight into domain of proteins is also shown in Fig. 4.

Six common genes of hypothyroidism were selected. Their nucleotide sequences are given in supplementary files. They were BLAST searched to see their homology. Basic information of all genes is given in Table 1. All genomes present in the modern biosphere have evolved from common ancestral genomes. Comparative genomics has enabled us to identify and study commonly derived processes which occur both in humans and model organisms. Comparative genomic studies helped us to identify and study commonly derived processes which occur both in humans and model organisms. Modeling human genetic diseases in these organisms requires the correct assignment of orthology. Orthologs are described as genes in differentt species that have originated from a single gene in the last shared ancestor, i.e., homology following speciation. Homologs that arise from gene duplications are termed paralogs, a term often applied to homologs within a genome. Thyroid stimulating hormone receptor (TSHR) TSHR gene has 74 orthologue in which 11 species are Primates which are given in the table below. Orthologous BLAST search results of all six genes are available in supplementary file. TSHR gene is present on the chromosome

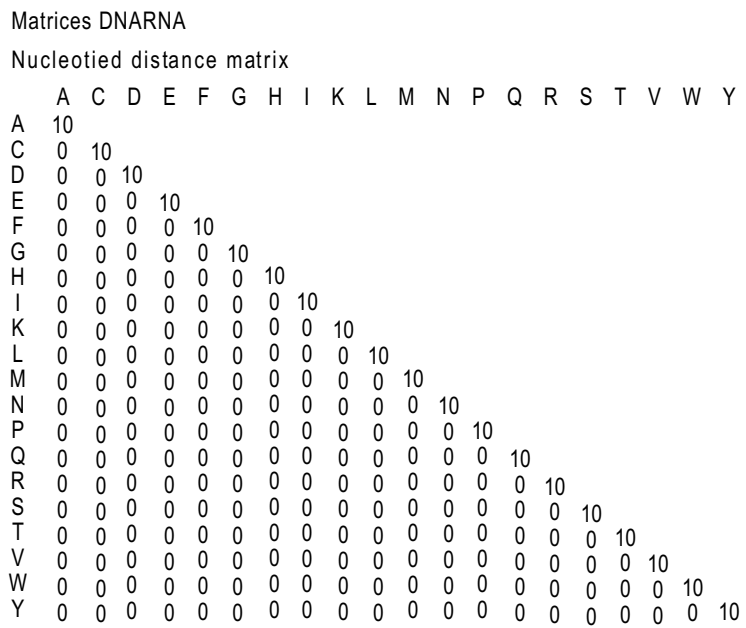

Fig. 3. Scoring matrix. 


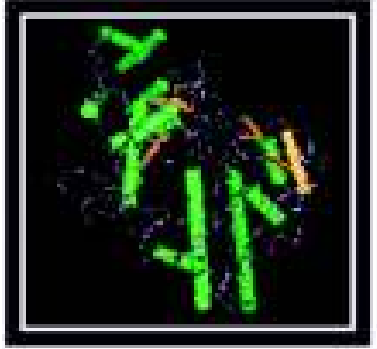

Protein of TPO gene

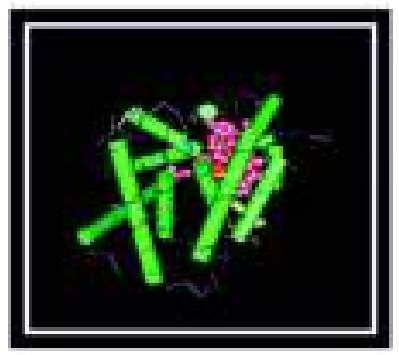

Protein of THRB gene

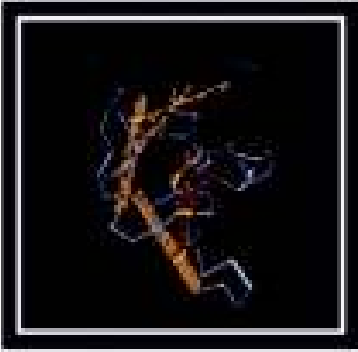

Protein of TG gene

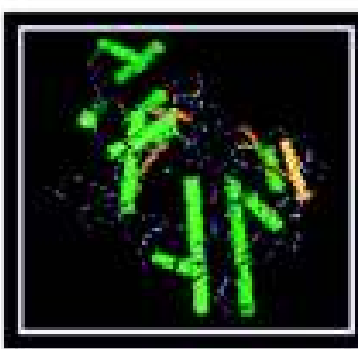

Protein of DUOX2 gene

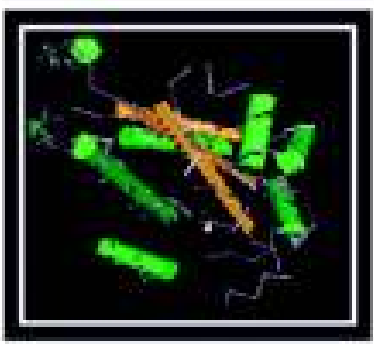

Protein of IYD gene

Fig. 4. Protein folding structure of TSHR, TPO, THRB, TG, IYD, DUOX2. no 14 and accession id of gene is KJ897710.1. Thyroid peroxidase (TPO) gene has 62 orthologues in which 11 Primates species. The chromosome on which TPO gene is located is chromosome no 2 and accession ID of the gene is DQ011222.1.THRB gene has 69 orthologues in which 11 species of primates. THRB gene is located on the chromosome no 3 and have accession ID which is KJ892275.1. Thyroglobulin TG gene has 68 orthologues in which 11 species of Primate. The accession Id of TG gene is BC054883.1 and located on the chromosome no 8. IYD (Iodotyrosine deiodinase) is a gene with the accession id of KJ900706.1 and present on chromosome no 6.IYD gene has 66 orthologues in which 11 species are Primates, given in the table below. DUOX2 has 72 orthologues in which 11 species are Primates which are given in the Table 1. DUOX2 is the gene which is present on the chromosome no 15 (Endo et al., 1995) and accession id of that gene is AF267981.1.

Improved technology in genetic surveys have already provided us with a lot of information on the genetic basis for 'normal' thyroid function, autoimmune thyroid disease and the influence of thyroid genes on clinical phenotypes. The need for larger sample sizes and collaboration between groups with access to large educational institutes has now been understood, and these studies will undoubtedly discover further genes. Furthermore, whole genome sequencing may provide more information as other types of genetic variation,

Table 1. Basic information of genes of hypothyroidism

\begin{tabular}{llllll}
\hline \hline $\begin{array}{l}\text { Official } \\
\text { symbol }\end{array}$ & Official full name & Primary source & Gene type & Chromosome no. & Also known as: \\
\hline TSHR & $\begin{array}{l}\text { Thyroid stimulating } \\
\text { hormone receptor }\end{array}$ & HGNC:HGNC:12373 & Protein coding & $\begin{array}{l}\text { Chr14,NC-000014.9 } \\
(80954989 . .81146302)\end{array}$ & LGR3;CHNG1;hTSR-I \\
THRB & $\begin{array}{l}\text { Thyroid hormone } \\
\text { receptor beta }\end{array}$ & HGNC:HGNC:1179 & Protein coding & $\begin{array}{l}\text { chromosome 3,NC-000003.12 } \\
(24117153 . .24495281, \\
\text { complement })\end{array}$ & $\begin{array}{l}\text { GRTH;PRTH;THR1 } \\
\text { ERBA2;NR1A2;THRB1 }\end{array}$ \\
TG & Thyroglobu-lin & HGNC:HGNC:11764 & Protein coding & $\begin{array}{l}\text { Chr 8,NC-000008.11 } \\
(132866943 . .133134902)\end{array}$ & TGN;AITD3 \\
TPO & Thyroid peroxidase & HGNC:HGNC:12015 & Protein coding & $\begin{array}{l}\text { Chr 2,NC-000002.12 } \\
(1413461 . .1542729)\end{array}$ & MSA;TPX;TDH2A \\
IYD & $\begin{array}{l}\text { Iodothyrosine } \\
\text { deiodinase }\end{array}$ & HGNC:HGNC:21071 & Protein coding & $\begin{array}{l}\text { Chr 6,NC-000006.12 } \\
(150368854 . .150404629)\end{array}$ & TDH4;IYD-1;DEHAL1; \\
DUOX2 & Dual oxidase2 & HGNC:HGNC:13273 & Protein coding & $\begin{array}{l}\text { Chr 15,NC-000015.10 } \\
(45092653 . .45114161)\end{array}$ & $\begin{array}{l}\text { TDH6;LNOX2; } \\
\text { THOX2;NOXEF2; }\end{array}$ \\
\hline \hline
\end{tabular}


such as copy number variants may also be found to play a role. In addition, influences on accessibility of genes to transcription may be at work. However, what we have already discovered has increased our understanding of normal thyroid hormone action and physiology, and we are starting to understand the complex origins of hypothyroid disease. For future work, need is to replicate the early findings presented above and perform functional studies to identify the true associations and the mechanisms behind them. These mechanisms will increase our understanding of thyroid physiology and identify therapeutic targets.

\section{Conclusion}

Bioinformatics of human diseases genes is a strategy, which combines nucleotide and protein sequences, enhanced versions of BLAST search, methods for motif analysis, and specialized databases, to the analysis of protein sequences encoded by positionally cloned human disease genes which resulted in the detection of a number of previously undetected, to our knowledge, conserved motifs and several predictions of disease gene functions. Most of the disease gene products show high significant similarity to proteins from the other species. Orthologous and conserved domain study helps us in understanding the disease gene products and changes in domain architecture.

Conflict of Interest. The authors declare no conflict of interest.

\section{References}

Andersson, M., Takkouche, B., Egli, I., Allen, H.E., deBenoist, B. 2005. Current global iodine status and progress over the last decade towards the elimination of iodine deficiency. Bulletin of World Health Organization, 83: 518-525.

Bakker, B., Bikker, H., Vulsma, T., deRandamie, J.S., Wiedijk, B.M., deVijlder, J.J. 2000. Two decades of screening for congenital hypothyroidism in The Netherlands. TPO gene mutations in total iodide organification defects. The Journal of Clinical Endocrinology and Metabolism, 85: 3708-3712.

Cangul, H., Aycan, Z., Olivera-Nappa, A., Saglam, H., Schoenmakers, N.A., Boelaert, K., Cetinkaya, S., Tarim, O., Bober, E., Darendeliler, F., Bas, V., Demir, K., Aydin, B.K., Kendall, M., Cole, T., Högler, W., Chatterjee, V.K., Barrett, T.G., Maher,
E.R. 2003. Thyroid dyshormonogenesis is mainly caused by TPO mutations in consanguineous community. Clinical Endocrinology, 79: 275-281.

DeVijlder, J.J.M., Dinsart, C., Libert, F., vanKessel, A.G., Bikker, H., Bolhuis, P.A., Vassart, G. 1988. Regional localization of the gene for thyroid peroxidase to human chromosome. Cytogenetic Cell Genetics, 47: 170-172.

Endo, Y., Onogi, S., Umeki, K. 1995. Regional localization of the gene for thyroid peroxidase to human chromosome $2 \mathrm{p} 25$ and mouse chromosome 12C. 1995. Genomics, 25: 760-761.

Engum, A., Bjøro, T., Mykletun, A., Dahl, A.A. 2002. An association between depression, anxiety and thyroid function- a clinical fact or an artefact. Acta Psychiatrica Scandinavica, 106: 27-34.

Goichot, B., Caron, P., Landron, F., Bouée, S. 2015. Clinical presentation of hyperthyroidism in a large representative sample of outpatients in France: relationships with age, aetiology and hormonal parameters. Clinical Endocrinology, 84: 445-451.

Hedberg, C.W., Fishbein, D.B., Janssen, R.S. 1990. An outbreak of thyrotoxicosis caused by the consumption of bovine thyroid gland in ground beef. The New England Journal of Medicine, 316: 993 998.

Hollowell, J.G., Staehling, N.W., Flanders, W.D. Serum, T.S.H. 2002. T(4), and thyroid antibodies in the United States population (1988 to 1994): National Health and Nutrition Examination Survey (NHANES-III). Journal of Clinical Endocrinology Metabolism, 87: 489-499.

Kimura, S., Hong, Y.S., Kotani, T., Ohtaki, S., Kikkawa, F. 1989. Structure of the human thyroid peroxidase gene: comparison and relationship to the human myeloperoxidase gene. Biochemistry, 28: 44814498.

Pilo, A., Iervasi, G., Vitek, F., Ferdeghini, M., Cazzuola, F., Bianchi, R. 1990. Thyroidal and peripheral production of 3,5,3'-triiodothyronine in humans by multi compartmental analysis. American Journal of Physics, 258: 715-726.

Surks, M.I., Ortiz, E., Daniels, G.H., Sawin, C.T., Col, N.F., Cobin, R.H., Franklyn, J.A., Hershman, J.M., Burman, K.D., Denke, M.A., Gorman, C., Cooper, R.S., Weissman, N.J. 2004. Sub clinical thyroid disease: Scientific review and guidelines for diagnosis and management. Journal of American 
Medicine Association, 291: 228-238.

Taurog, A., Howells, E.M. 2000. Hormone Synthesis:

Thyroid Iodine Metabolism. pp. 61-84, $8^{\text {th }}$ edition, Williams \& Wilkins, Philadelpia.

Vetter, M.L., Kaul, S., Iqbal, N. 2008. Tyrosine kinase inhibitors and the thyroid as both an unintended and an intented target. Endocrinology Practice,
14: 618-624.

Wekking, E.M., Appelhof, B.C., Fliers, E., Schene, A.H., Huyser, J., Tijssen, J.G., Wiersinga, W.M. 2005. Cognitive functioning and well-being in euthyroid patients on thyroxine replacement therapy for primary hypothyroidism. European Journal of Endocrinology, 153: 747-753. 